\title{
Territorio y ganadería en la Patagonia Argentina: desertificación y rentabilidad en la Meseta Central de Santa Cruz
}

\author{
LARRY ANDRADE*
}

\begin{abstract}
The paper briefly analyses the characteristics of desertification in Austral Patagony, particularly in the province of Santa Cruz, Argentina. A reference is presented about the spreading of sheep stock in Santa Cruz lands in the late $19^{\text {th }}$ century, and more intensively in the beginnings of $20^{\text {th }}$ century, as well as the impact it caused, particularly by giving rise to an erosion process (desertification), due to excesive sheperding in the natural pasture. Some references are given about the livestock farming sector and its participation within the geographic gross product (PBG, producto bruto geográfico in spanish) of Santa Cruz. Finally, the paper offers a comparative analysis of the number of animals that producers consider they can hold in their fields and the number of animals the National Farming and Livestock Institute (INTA, Instituto Nacional de Tecnología Agropecuaria in spanish), based on pasture relieving, considers it could bold.
\end{abstract}

Keywords: Austral Patagony, desertification, $P B G$, oversheperding-stock.

\section{Resumen}

El artículo revisa brevemente las características que asume el proceso de desertificación en la Patagonia Austral, con énfasis en la provincia de Santa Cruz, Argentina. Se hacen referencias a la ocupación del territorio santacruceño por el ganado ovino, a fines del siglo XIX y con más intensidad a principios del siglo xx, y el impacto que ello conllevó, especialmente el desencadenamiento de un proceso erosivo (desertificación), producido básicamente por el pastoreo constante (sobrepastoreo) del pastizal natural. También se encuentran referencias al sector ganadero y su participación en el producto bruto geográfico ( $\mathrm{PBG}$ ) de Santa Cruz y, hacia el final, un análisis comparativo de la cantidad de animales que el productor estima que puede sostener en su campo y la cantidad que el Instituto Nacional de Tecnología Agropecuaria (INTA), con base en relevamientos de pastizal, calcula que podría mantener.

Palabras clave: Patagonia Austral, desertificación, PBG, stock de sobrepastoreo.

*Universidad Nacional de la Patagonia Austral. Correo-e: psanjulian@prodigy.net.mx 


\section{Introducción ${ }^{1}$}

Este artículo comprende una breve revisión en torno a la problemática de la desertificación y su relación con la explotación extensiva de ovinos, así como algunas consideraciones acerca del stock y la evolución de la participación del subsector ganadero en el producto bruto geográfico ( $\mathrm{PBG}$ ) provincial. El texto incluye algunas reflexiones en torno a la relación entre ganadería, territorio y desertificación.

Lo que a principios del siglo $\mathrm{xx}$ fue una actividad pionera $\mathrm{y}$ altamente rentable en el poblamiento del territorio santacruceño, con el correr de los años devino en una crisis cuya manifestación ha sido el cierre de más de quinientos establecimientos ganaderos. ¿Qué ocurrió en ese tiempo para llegar a tal desenlace? Cuando inicié la investigación, la pregunta que orientó las primeras búsquedas partía de una interrogante que se formulaban profesionales de distintos organismos técnico-agropecuarios: ¿̇por qué no adoptan tecnología los productores extensivos de ovinos? A partir de allí se abría un universo de situaciones que podrían resumirse en una sola y gran cuestión: ¿cómo vislumbraban los productores la crisis de la ganadería extensiva de ovinos en sus campos y qué relación mantenía esta crisis con la falta de adopción de tecnología de manejo extensivo? Esta pregunta era independiente de la percepción que los ganaderos tuvieran de la tecnología en sí misma, e interesaba conocer cómo se relacionaba el productor con el recurso natural que explotaba -es decir, cómo lo 'veía'- y qué evaluación hacía de su práctica productiva. Este análisis se realizó en función de grupos de productores, los cuales fueron definidos en una tipología desarrollada de modo extenso en la investigación.

El trabajo pretende ser una mirada que amplíe la visión propia del ganadero en torno a la rentabilidad de las explotaciones y considere dicha visión como el criterio más importante, en estudios que procuran explicar la permanencia o el abandono de

${ }^{1}$ La investigación "Representaciones sociales de la desertificación. El caso de los productores ovino-extensivos en la zona centro de la meseta central, Provincia de Santa Cruz", de la cual este artículo es producto, fue posible gracias al aporte financiero de la Universidad Nacional de la Patagonia Austral, el Instituto Nacional de Tecnología Agropecuaria (Estación Experimental Agropecuaria Río Gallegos, Santa Cruz) y el Proyecto de Prevención y Control de la Desertificación para el Desarrollo Sostenible de la Patagonia-Convenio INTA/GTZ (Cooperación Técnica Alemana). El director fue Guillermo Neiman (CEIL/CONICET). Algunos conceptos de este apartado fueron presentados en Andrade (1999b). 
las explotaciones. Desde esta propuesta, resultaba una tarea ineludible en el abordaje de ese contexto la comprensión de las prácticas sociales de producción en esos espacios y de los significados asociados a ellas, en tanto 'lo real' cobra importancia a partir de la significación e interés que reviste para el individuo (Weber, 1993). ${ }^{2}$ Un análisis detallado del discurso de los productores en torno a la problemática está descrita con cierto en detalle en otro lugar Andrade (1999a y 2002). ${ }^{3}$ La ubicación relativa de la provincia y zona de estudio puede verse en el mapa 1.

Existe un consenso generalizado entre los expertos y los diversos organismos técnicos provinciales, nacionales e internacionales acerca de que el fenómeno conocido como desertificación se debe, al menos en Patagonia, al sobreuso de los suelos que, asociado con factores climáticos, llevó a un agotamiento del recurso natural, base de la explotación extensiva del ganado ovino por más de cien años.

La cuestión crucial, desde mi óptica, era tener una aproximación confiable a la visión que los productores implicados en este proceso tenían de la situación del ecosistema y a qué causa atribuían la merma en el número de animales y, consecuentemente, en la producción de lana, a la vez que interesaba conocer las razones que aducían ante la imposibilidad de repoblar el campo con nuevo ganado.

Lo que procuro dejar en claro es que la situación del ecosistema es un síntoma en el ambiente natural de una crisis más grande y extendida, originada en la baja casi constante de la rentabilidad que ha impactado la ganancia de estos productores, desde el fin de la Primera Guerra Mundial; lo que llevó a muchos de ellos a recargar sus campos para compensar las pérdidas. Es decir, es el efecto visible de la crisis de un sistema social de producción erigido en torno a la explotación extensiva de ovinos.

\footnotetext{
2 Recuperando esta idea, a mediados de la década de los años cincuenta, Alfred Schutz postuló una premisa que puede considerarse básica para la sociología comprensiva: "el campo de observación del científico social, el mundo social, no es esencialmente inestructurado. Tiene un sentido particular y una estructura de significatividades para los seres humanos que viven, piensan y actúan dentro de él (...) éstos han preseleccionado y preinterpretado este mundo mediante construcciones de sentido común acerca de la realidad cotidiana. Esos objetos de pensamiento determinan su conducta, definen los objetivos de su acción, los medios disponibles para alcanzarlos (...) los ayudan a orientarse dentro de su medio natural y sociocultural y a relacionarse con él" (Schutz, 1995: $37)$.
}

${ }^{3}$ Pueden solicitarse a: psanjulian@prodigy.net.mx 


\section{Mapa 1}

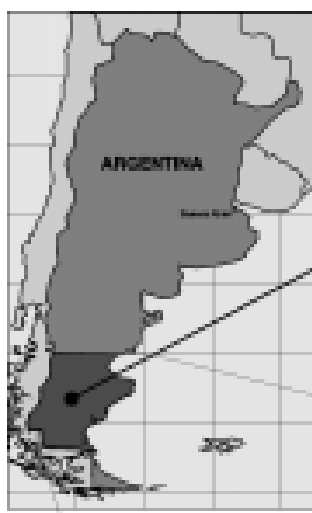

a)

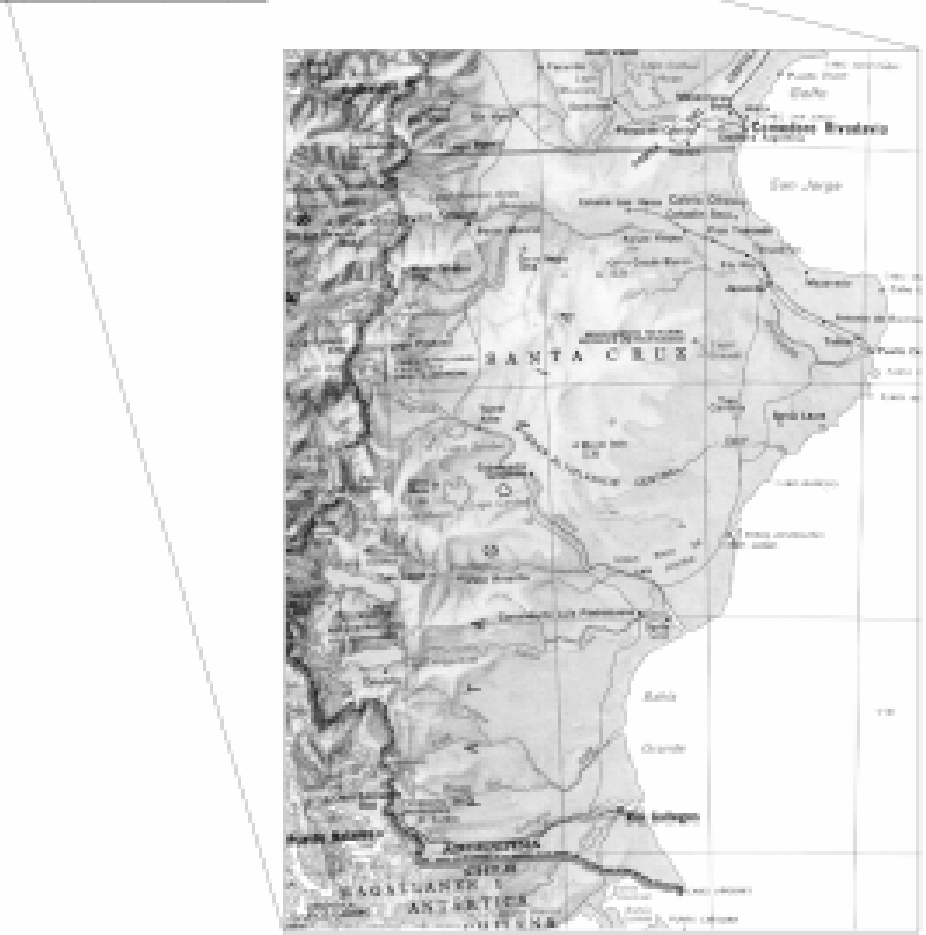

b)

Fuente: Atlas Barsa, 1982, Encyclopaedia Britannica, Inc. 


\section{Breves consideraciones metodológicas}

\subsection{Datos}

\subsubsection{Datos secundarios}

De los dos tipos de datos que utilicé, primarios y secundarios, en la selección y análisis de estos últimos fue prioritaria la bibliografía específicamente elaborada y orientada a la problemática del área de estudio. Esto tiene su razón de ser ya que el proceso erosivo asociado con pastoreo ovino, característico de la Patagonia Austral en general, no registra antecedentes en ecosistemas áridos fríos del mundo (INTA, 1997).

De esta manera, la primera información con la que me acerqué al problema provino de la lectura de informes técnicos, en su gran mayoría del Instituto Nacional de Tecnología Agropecuaria (INTA), del Consejo Agrario Provincial (CAP) y de otros organismos como el Consejo Federal de Inversiones (CFI), así como de las publicaciones periódicas de estas instituciones y otras dedicadas a la problemática agropecuaria.

Mención aparte merece el trabajo con fuentes históricas originadas a partir de investigaciones desarrollados en esa provincia; tal es el caso del libro de Horacio Raúl Lafuente (1981); los artículos de María E. Cepparo de Grosso (1986) y el libro de Elsa Barbería (1995) sobre la ocupación del territorio santacruceño y documentos inéditos, provistos por la misma autora. La revisión de estos trabajos se constituyó en una referencia fundamental con el fin de presentar y analizar las bases sobre las que se acentó y consolidó el método de producción extensiva de ovinos en Patagonia. Otra fuente importante de información fueron los Censos Nacionales de Población y Agropecuarios y la Encuesta Nacional Agropecuaria aplicada en distintos años.

\subsubsection{Datos primarios}

\subsubsection{La encuesta}

La encuesta aplicada a los productores se tomó en un lapso de tres meses (marzo, abril y mayo de 1998), y se contó con la colaboración del ingeniero agrónomo Héctor Espina y del técnico agropecuario Roberto Álvarez para la concreción de algunas de ellas, especialmente en la realización del pre-test. Participaron, 
además, en distintas circunstancias, los biólogos Leopoldo Montes y Gabriel Oliva junto con el ingeniero agrónomo Pablo Bore1li, todos de la Estación Experimental Agropecuaria INTA, Río Gallegos, quienes realizaron valiosas sugerencias y aportes que permitieron un mayor ajuste del cuestionario.

Previo a la aplicación definitiva de la encuesta, realicé con ellos una última revisión del instrumento y los proveí de un instructivo preparado especialmente para la ocasión, donde se explicaban cada una de las preguntas y el fin al que se dirigían, esperando de ese modo contribuir a la resolución de posibles dudas o malos entendidos que pudieran surgir durante el trabajo de campo.

La encuesta finalmente aplicada constó de 87 items, que cubrían las siguientes dimensiones:

i. Datos socio-demográficos del productor y su familia;

ii. Condiciones naturales de la explotación;

iii. Alternativas productivas para esa explotación;

iv. Características productivas de la explotación;

v. Forma de tenencia y manejo de la explotación;

vi. Comercialización de la producción;

vii. Posibilidades de uso de tecnología en la explotación;

viii. Aspectos culturales del productor.

Concluida la etapa de recolección de datos mediante un cuestionario estandarizado, se obtuvieron 39 encuestas, de las cuales 25 se aplicaron a productores del área de trabajo y 14 fuera de la misma, puesto que en la zona delimitada para el estudio no fue posible, en el lapso previsto para su aplicación, encontrar más que ese número de productores. El número de 39 productores no responde a ningún criterio previo para su obtención: fueron los encontrados hasta el día antes de que concluyera el tiempo previsto para esa tarea.

Los 14 productores encuestados fuera del área comparten la situación en cuanto a grados de desertificación y crisis de rentabilidad que envuelve a los primeros. Por tal motivo se integran a la muestra como parte de la misma totalidad. ${ }^{4}$

${ }^{4}$ La obtención de los casos para la encuesta no se realizó por muestreo probabilístico, dado que el área de estudio incluía 69 establecimientos y era de interés de los organismos involucrados en la investigación relevarlos a todos. Además de ser un número relativamente pequeño para realizar análisis estadístico, se agregaba el hecho de que algunos de ellos eran propiedad de una sola persona. Algunas de esas explotaciones habían sido abandonadas tiempo atrás, incluso antes de las grandes catástrofes climáticas de 1981 y 1984. 


\subsubsection{Las entrevistas}

Realicé entrevistas con productores del área de Gobernador Gregores y también algunas con representantes de instituciones que aparecían directamente vinculadas con el quehacer agropecuario en la zona, como son el director la Escuela Agropecuaria núm. 2 de Gobernador Gregores, el Delegado del Consejo Agrario Provincial (CAP) en esa localidad, el presidente del CAP, algunos dirigentes de la Sociedad Rural de Puerto San Julián y de Gobernador Gregores. Entre 1996 y 1998 entrevisté a 24 productores y funcionarios, algunos de los cuales fueron entrevistados de nueva cuenta posteriormente. Las entrevistas giraron en torno a cinco temas centrales para el estudio:

i. Historia de la explotación;

ii. Historia personal en la explotación;

iii. Estado productivo actual de la explotación;

iv. Causas que advierte como desencadenantes de la crisis;

v. Factores en los que deposita su confianza con el fin de hacer posible un eventual despegue productivo.

\subsubsection{Generación de una tipología de productores}

Con el objeto de lograr una aproximación lo más ajustada posible a la percepción de la desertificación (básicamente con información provista por las entrevistas y algunas preguntas abiertas de la encuesta), asumí que la elaboración de una tipología sería de utilidad en ese acercamiento y, por otro lado, permitiría un manejo ordenado de la cantidad de datos que disponía a partir de la aplicación de la encuesta.

Con la idea de describir de la forma más precisa posible las diferencias entre productores, opté por generar una tipología que, precisamente, según Barton (1955: 213), tiene por objeto reducir un espacio de atributos relativamente complejo, precisamente aquel espacio multidimensional que aparecía como horizonte del análisis de información provista por la encuesta.

Las tipologías, según Hughes, Griffon y Bouveyron (1978: $105)$, están orientadas por un fin, cuyo "criterio fundamental es poner de relieve las anomalías de distribución de una población en relación con diversas variables consideradas simultáneamente y sobre un plano de igualdad"; lo cual es complementado por Barton cuando expresa que además de la función de organización de conjuntos complejos de datos, "permiten analizar las com- 
ponentes de dichas variables con objeto de determinar el papel que cada una de ellas desempeña” (Barton, 1955: 214).

Al hablar de tipología estamos hablando de grupos de tipos, esto es, de un conjunto de variables características de un número determinado de personas y que se diferencian relativamente de otro grupo distinto de personas. El interés por resaltar la diferencia de dichas carácterísticas es con el fin de distinguir cómo la mayor o menor presencia de éstas puede influir en la explicación de la variable dependiente.

El criterio que guió la construcción de la tipología fue la agrupación de las personas con características homogéneas en un tipo, bajo el supuesto de homogeneidad relativa, puesto que existe igualmente cierta heterogeneidad en el grupo, pero que en su conjunto esos casos presentan mayor afinidad entre sí que con los de otro tipo. De este modo, la tipología servía con fines de generalización de los resultados a partir del análisis de casos particulares, posteriormente agrupados en tipos y de comparación entre éstos.

La revisión de investigaciones donde se habían empleado tipologías no permitía encontrar criterios que pudiera aplicar directamente en mi trabajo, con la excepción de dos trabajos, uno producido por el INTA (s.f.) y otro por el LUDEPA (1992), que abordaban elementos para una tipología de productores patagónicos.

A partir de esta constatación y con la información aportada por la encuesta, generé una tipología de productores. Con la información obtenida en las entrevistas profundicé al interior de cada grupo e indagué en lo que más interesaba: cómo ven y a qué adjudican los productores la crisis actual del campo santacruceño en la zona en estudio, y cómo avizoran una eventual salida productiva de sus explotaciones.

El criterio por el cual se definieron los tipos fue a partir de la diferencia entre las variables ingresos del establecimiento, que surgió con un peso relativo importante en algunos análisis de correlaciones, y gastos del establecimiento en el último año como criterio de demarcación, a partir de los cuales se generaron tres grupos de productores: Grupo (I) nivel de sobrevivencia (con un saldo negativo entre gastos e ingresos); Grupo (II) en transición (el gasto y el ingreso es igual), y Grupo (III) sostenibles económicamente (el saldo entre gastos e ingresos es positivo). En el primer grupo se ubican 23 establecimientos, en el segundo 5 y en el tercero 11. 


\section{Ubicación y descripción de la región patagónica argentina ${ }^{5}$}

La región patagónica argentina, comprendida entre los paralelos $36^{\circ}$ y $55^{\circ}$ de latitud sur, cubre un área de $780 \mathrm{mil} \mathrm{km}^{2}$ y representa aproximadamente un tercio de la superficie continental del país. En las cinco provincias que la integran -Neuquén, Río Negro, Chubut, Santa Cruz y Tierra del Fuego- habitan un millón quinientos mil personas, siendo la densidad poblacional de 1.9 $\mathrm{hab} / \mathrm{km}^{2}$. Si bien esta extensa región presenta diferentes áreas ecológicas, la mayor parte de ella puede ser caracterizada como una región árida-semiárida con precipitaciones que van de los 100 a los $300 \mathrm{~mm}$ anuales. Los vientos -constantes gran parte del año- son predominantes del sector oeste y con intensidades de moderados a fuertes. Los suelos son sueltos, con reducida cobertura vegetal de bajo valor forrajero.

El sector agropecuario, fuera de los valles irrigados, involucra alrededor de 12 mil productores. La ganadería extensiva ovina, bovina y caprina es la principal actividad, y se produce lana, carne, pelo y pieles.

$\mathrm{Al}$ desencadenamiento de un proceso erosivo por la introducción masiva de especies foráneas se suman la extracción de especies leñosas (arbustos y árboles) para combustible, la explotación petrolera y minera, el mal uso del agua (salinización y revenimiento), y los incendios de campos y bosques.

\section{Desertificación en Santa Cruz: breve estado de la discusión}

Se hará aquí una breve presentación de la problemática asociada con la crisis de la ganadería extensiva de ovinos, esto es, la desertificación y las distintas formas de abordar este fenómeno. Una definición breve y difundida del fenómeno conocido como desertificación es la propuesta por Bertolani: "la desertificación es la extensión de las condiciones de desierto como resultado del impacto humano, en ecosistemas de regiones áridas, semiáridas y sub-húmedas [...] el no control del fenómeno provocaría no sólo un avance en las condiciones de desierto sino, y como consecuencia de ello, una menor productividad de los ecosistemas" (Bertolani, 1989).

En otro trabajo se la define del siguiente modo: "la desertificación es el proceso de empobrecimiento de los ecosistemas

${ }^{5}$ Basado en información obtenida en: INTA (s.f.). 
áridos, semiáridos y algunos subhúmedos, a causa del impacto combinado de las actividades del hombre y la sequía" (Dregne, 1976, en Peralta, 1992), o también, "la desertificación es un proceso natural o inducido por el hombre, de irreversible cambio de suelo y vegetación de zonas áridas en la dirección de la aridificación y disminución de la productividad biológica, la cual en casos extremos, puede orientarse hacia la total destrucción del potencial biológico y conversión de la tierra hacia un desierto" (Peralta, 1992).

Estudios llevados a cabo en el área en estudio encuentran que "la desertificación observada parece deberse a: 1) intensificación por sobrepastoreo de los procesos erosivos en áreas naturalmente propensas; 2) sobrespatoreo continuo sobre comunidades frágiles, y 3) sobreexplotación de comunidades de arbustos” (Espina, 1994: 9).

Rapp, Acosta y Ayerza (1988) aportan elementos que permiten una comprensión más acabada del fenómeno en Santa Cruz: "haciendo un análisis somero de los factores que inciden para acelerar la degradación del ambiente, corresponde señalar que: 1) la vegetación típica es de estepa y semidesierto, rala y con mucho suelo descubierto; 2) los vientos se orientan en forma dominante de la cordillera hacia la costa (oeste-este) $;^{6} 3$ ) las precipitaciones son sumamente escasas; 4) las bajas temperaturas no permiten el desarrollo de una microflora y fauna del suelo, que degrade la poca materia orgánica incorporada en los ácidos húmicos que coadyuven a la formación de un horizonte superficial resistente; 5) el material del suelo carece de elementos finos por la acción del viento; 6) sobre todo este panorama tan desalentador, el sobrepastoreo y pisoteo son las dominantes de una situación muy difícil de revertir" (Rapp et al., 1988: 155).

La opinión del ingeniero agrónomo Borelli, quien expresaba hace unos años que se ha "cumplido un siglo de ganadería en Santa Cruz y todavía no se conoce cuánto pasto producen los pastizales, qué especies son preferidas, cuál es la resistencia al

${ }^{6}$ Un factor constante y de permanente acción sobre el suelo es el viento, que sopla con fuerte intensidad la mayor parte del año. En tal sentido, Rapp, et al. (1998) lo mencionan como uno de los factores que impactan en la rentabilidad de las explotaciones y en el desmejoramiento del estado del ecosistema: "las características climáticas altamente agresivas, que sumadas al mal manejo que desde los inicios de la monocultura ovina se ha dado a estas tierras, acarrean una disminución progresiva de la rentabilidad", y luego agregan que "la causa principal de la erosión es la sobrecarga animal provocada por distintos factores tales como inestabilidad económica del país, desconocimiento del manejo racional y falta de precio adecuado para la carne ovina que orientó al productor a la mayor producción de lana” (Rapp et al., 1988: 156). 
pastoreo y, en consecuencia, cuánto pasto se puede cosechar a través de los animales. Este lamentable desconocimiento ha favorecido el proceso generalizado de sobrepastoreo y degradación de los campos naturales (...) la carga animal define la relación planta-animal y los ingresos económicos a corto y largo plazo de la empresa ganadera" (Borelli, s.f.). Este autor refuerza lo ya señalado y apunta hacia los efectos producidos en la explotación y en la rentabilidad esperada por el productor.

En algunas de las expresiones anteriores se menciona el sobrepastoreo como un factor decisivo en el desencadenamiento y profundización del proceso de desertificación. A tal fin, Panigatti lo define como aquella acción que "produce degradación de la vegetación natural o cultivada y consecuentemente una denudación del suelo. En muchos casos el pisoteo en condiciones desfavorables aumenta la acción negativa, compactando los suelos finos o pesados, con la consecuente reducción de la capacidad de infiltración" (Panigatti, 1988: 48).

Un estudio reciente identifica factores estructurales y coyunturales en el estancamiento y crisis del sistema ovino patagónico. Entre los primeros encuentra "la baja inversión en tecnología y el proceso de desertificación provocado por la explotación intensiva (sobrecarga ovina), y entre los segundos la evolución negativa de los precios internacionales de la lana" (LUDEPA, 1992: 27).

Quizá sea oportuno realizar una aclaración a este señalamiento o, mejor aún, vincular de otro modo estos elementos: según entiendo, el bajo precio de la lana es un factor considerado como coyuntural desde hace ya varios años, especialmente por parte de los productores. Sin embargo, existen estudios no tan recientes (por ejemplo, FLA, 1986), que muestran que el bajo precio que se paga en el mercado internacional por la lana es una constante, por lo menos desde el fin de la Segunda Guerra Mundial, y responde, entre otras situaciones, a la producción masiva de sustitutos de la lana.

Desde esta visión, el bajo precio de la lana es un factor estructural. La baja inversión en tecnología existe, y lo que hace falta es profundizar en los motivos o razones de tal actitud. Esta situación se agrava para los productores de la Patagonia Austral, donde al de por sí bajo precio para lanas de calidad en el mercado internacional, se agrega como elemento que reduce el valor del producto la suciedad que presenta su producción, afectando notablemente el rinde (es decir, la proporción de lana limpia por 
kilo de lana obtenida) y, con ello, el precio final obtenido por el ganadero.

En las menciones precedentes, es notable el centramiento del análisis en aspectos básicamente técnicos, soslayando el rol fundamental que tienen (y tuvieron) los productores y los organismos técnicos en el desencadenamiento del proceso erosivo asociado a la ganadería extensiva de ovinos. Es decir: no basta con saber técnicamente el estado del pastizal y su capacidad receptiva, sino también es necesario conocer los criterios y elementos que el productor valora en el momento de tomar la decisión de introducir o quitar animales de su campo.

Los departamentos de la zona centro ubicados sobre la costa y los ubicados sobre la meseta central son los más expuestos y afectados por la desertificación: "Magallanes, Río Chico y Corpen Aike sufren con mayor intensidad un notable y paulatino descenso de la carga ovina debido a graves problemas de erosión. Se combina el proceso erosivo natural por fuertes vientos con un manejo inadecuado, desorganizado y sin descanso de las pasturas debido a la falta de campos de uso estacional, desencadenando la disminución de la receptividad de los mismos. Esta situación pudo haber sido alentada por ganancias momentáneas y por el desconocimiento de la receptividad, llevando a una caída espectacular de la productividad por hectárea y a no poder sostener ya las cargas animales de comienzos de siglo" (Cepparo de Grosso, 1986: 92; Borelli, s.f.).

Una de las consecuencias más notorias del avance del proceso erosivo es el abandono de establecimientos. Al respecto, Barbería expresa que la mayoría de los "establecimientos abandonados y no rentables se ubican en la meseta central, al norte del Río Santa Cruz, zona que cuenta con los terrenos de menor calidad, menor superficie y menores inversiones. Por otra parte, un sector importante de los mismos ha sido cubierto de ceniza volcánica a raíz de la erupción del Volcán Hudson -agosto de 1991- [...]” (Barbería, 1995: 277).

Y esa es precisamente el área donde este trabajo indagó, puesto que a pesar de que fue la última zona en ocuparse (Barbería, 1995) y ser tierras de menor calidad que las del sur y la cordillera, sus ocupantes disfrutaron, aunque por poco tiempo, del auge del lanar y los altos precios obtenidos por la venta de lana. En los años 90 fue la zona más afectada por la desertificación en la que aún persisten algunos pobladores; la zona norte fue prácticamente abandonada a principios de esa década. 


\section{Sobrepastoreo y abandono de establecimientos}

Un dato significativo respecto del desencadenamiento de la crisis actual señala que a principios de siglo, cuando los peritos estatales relevaron el área de estudio con el fin de realizar la división catastral, sobreestimaron la receptividad de los suelos. Aun con las recomendaciones de equilibrar zonas altas y zonas bajas (veranadas e invernadas), estimaron una carga de 0.4 ovino/ha [aproximadamente 8 mil ovejas en 20 mil ha] (Espina, 1994: 10). ${ }^{7}$ Según los organismos técnicos que trabajan en la provincia, esta era una carga que muy pocas áreas ecológicas podían resistir entonces y que algunos lugares excepcionalmente aptos podrían resistir hoy.

Respecto del manejo de ovinos en el pastizal natural, existen dos menciones significativas, realizadas cuando la explotación extensiva de ovinos iniciaba su ciclo de mayor prosperidad y la adopción de políticas tendentes a lograr un manejo razonable del recurso natural era posible y necesaria. La primera pertenece a Bailey Willis, quien expresaba en 1914 lo siguiente: "en verano e invierno, año tras año, las ovejas se apacientan en un mismo prado; las plantas comestibles apenas tienen oportunidad para desarrollarse o propagarse, mientras las hierbas nocivas se multiplican por doquier. El gobierno debería cuanto antes tomar posesión de las dehesas públicas a fin de tenerlas bajo su cuidado y de evitar el detrimento y hasta la destrucción de los pastos [...] los rebaños debieran trashumar de estación en estación de manera que pudieran pacer en dehesas donde abundan la hierba y el agua y volver a dehesas más saludables" (citado en Peralta, 1992: 17).

La segunda, recuperada por Barbería, es la opinión de Clemente Onelli, quien en 1904 expresaba que "los resultados son negativos si se deja pastar en una determinada extensión un número excesivo de animales, entonces el campo se destruye, y para ser utilizado necesita a veces cinco o seis años de descanso" (citado en Barbería, 1995: 117).

${ }^{7}$ Sobre estos datos, la información es en apariencia variable, según podemos apreciar en Oliva, Rial y González (s.f.). Veamos el siguiente párrafo: "La subdivisión catastral se realizó de acuerdo a criterios geométricos, sin ninguna consideración acerca del balance invernada-veranada en los campos con mesetas altas, de la localización de aguadas o de la receptividad de cada sitio [...] se recomendaron cargas de alrededor de 0.2 equivalente ovino por ha $(0.2 \mathrm{Eo} / \mathrm{ha})$ )" (esta recomendación equivale a 4 mil ovinos en 20 mil ha). Sin embargo, prosiguen los autores: "hacía 1937, momento de máximo stock ovino, la carga promedio era de $0.45 \mathrm{EO} / \mathrm{ha”} \mathrm{(pp.} \mathrm{3-4).} \mathrm{Esta} \mathrm{carga} \mathrm{promedio} \mathrm{prác-}$ ticamente coincide con la señalada por Espina para el momento de la división catastral. Esto significa que si la recomendación fue de 0.2 ovino/ha, al mantener un promedio de $0.45 /$ ha, el efecto sobre el pastizal fue devastador. 
Las consecuencias de la falta de consideración de las situaciones a las que estas advertencias aludían son hoy visibles en la meseta central de Santa Cruz. Los daños en el pastizal natural son notables, especialmente en las explotaciones ubicadas en el centro y norte de Santa Cruz y en el noroeste de la Patagonia, donde la trashumancia es todavía una práctica vigente entre pequeños propietarios de ganado.

Al respecto, en un estudio realizado en Neuquén sobre hábitos culturales de ganaderos trashumantes encuentra "limitaciones estructurales" para el desarrollo sostenible de la producción; "dichas limitaciones se refieren a las dificultades para acceder a aquellos elementos que permiten un manejo ganadero mejor y más adecuado a sus necesidades (posibilidades de descanso y rotación en las veranadas, delimitación y/o apotreramiento de campos, mayor disponibilidad de aguadas, implementación de pasturas y/o complementación forrajera, diversos tipos de mejoras para el control de la erosión, mejoramiento animal, etc.)" (Bendini y Nogués, 1992: 18).

Si bien el contexto ecológico y social de Neuquén, por lo menos el de la ganadería trashumante, es diferente al de los productores de la meseta central, sí tiene importancia la mención en lo estrictamente relacionado con la capacidad financiera de los productores: si no tienen solvencia para realizar inversiones y mejoras, se ven obligados a repetir el ciclo de producción prácticamente sin cambios, con las consecuencias que ello conlleva. ${ }^{8}$

Según especialistas del INTA Santa Cruz, si el sobrepastoreo es constante, al cabo de algunos años se agota el recurso natural y no existe posibilidad de repoblamiento en el corto o mediano plazos. La mayoría de la vegetación de climas áridos fríos es perenne, con la excepción de algunas anuales de efímera existencia. Esto significa que mientras resisten las inclemencias climáticas, el pastoreo y el pisoteo sobreviven; una vez muertas, no hay posibilidad de repoblamiento vegetal.

El no tener una adecuada óptica de la situación y de sus factores desencadenantes o al adjudicar la causa de la crisis a las variaciones climáticas ha llevado a muchos productores a seguir manejando el campo como lo hacían tradicionalmente. En este

\footnotetext{
${ }^{8}$ Los mismos autores señalan que: "la persistencia de una forma de vida y producción no ajustada a los nuevos parámetros de la industria y el desarrollo nacional permite una acentuación del proceso paulatino de erosión por las prácticas repetitivas en cada ciclo, sin posibilidad de descarga y por la imposibilidad de disminuir el tamaño de los rodeos sin afectar la seguridad de un ingreso mínimo de las propias unidades domésticas de producción” (Bendini y Nogués, 1992: 19).
} 
contexto, Castro (s.f.) opina que "cuando un proceso de erosión se ha puesto en marcha, ya desde su calificación como 'leve' está afectando la producción forrajera de la pradera natural. El ganadero de la región generalmente resta importancia al fenómeno, porque no se da cuenta del mismo o porque atribuye la merma en la producción a la escasez de precipitaciones" (Castro, s.f.; también Barbería, 1994).

La crisis en el sector pecuario se asienta básicamente en la caída de la explotación extensiva de ovinos en casi todo el territorio santacruceño, con excepción de la zona sur y la zona cordillerana, donde hay grandes establecimientos en producción y algunos medianos en mejores suelos.

La combinación de baja inversión, poca o ninguna incorporación de tecnología, forma de tenencia precaria y uso inadecuado del suelo disminuyen aún más el ingreso obtenido por la venta de lana, en un mercado con precios de por sí deprimidos. Esta conjugación negativa de factores causó en 1997 el cierre de 500 establecimientos, según expresó a mediados de ese año el presidente de Federación de Instituciones Agropecuarias Santacruceñas (FIAS).

Para el año 1994, las estimaciones señalaban que sobre 1,261 establecimientos en la provincia (datos del año 1991), más de trescientos, casi exclusivamente orientados a la explotación ganadera extensiva, fueron abandonados y alrededor de setecientos no cubrían los costos operativos (Barbería, 1994). Muchos de los setecientos establecimientos deficitarios probablemente pasaron a engrosar la lista de quinientos cerrados en 1997.

\section{La ganadería y su participación en el producto bruto provincial}

Estudios realizados por la Universidad Federal de la Patagonia Austral y la Universidad de Buenos Aires dan cuenta de algunos elementos útiles para comprender la magnitud de la crisis: "existe un estancamiento en el sector rural de más de 20 años. La población rural registra una caída entre 1980 y 1991 del 19\%. Las existencias ovinas entre 1977 y 1992 cayeron en un 47\%. Las regiones más afectadas son las regiones centro y norte [...] disminuyó la población que habita los establecimientos (al disminuir el número de establecimientos rentables) en un $29 \%$ entre 1988 y 1992 y el número de trabajadores permanentes cayó en un 39\%" (UFPA/UBA/MTSS, 1994: 35 y ss.; UFPA/UBA/MTSS, 1995c). 
Esta situación impacta directamente en los núcleos urbanos de los departamentos donde esta explotación se asienta, especialmente Puerto Deseado y Puerto San Julián, ambas sobre la costa atlántica al norte y centro de la provincia, respectivamente. Estas ciudades sustentaron su crecimiento "en la explotación ovino-extensiva y en la exportación de lana y posteriormente en la venta de animales para las graserías y los frigoríficos. La crisis estructural de los setenta los afectó. Crecieron menos que otras regiones de la Provincia (menos del 20\% cada una)" (UFPA/UBA/ MTSS, 1995a: 10).

Un informe de la UFPA señalaba a principios de los años 90 la magnitud de esta crisis, mostrando que la participación por sectores en el producto bruto geográfico $(\mathrm{PBG})^{9}$ de "Agricultura, Ganadería, Caza, Pesca y Silvicultura (AGYP) pasó en 1970=15.7\% a $1980=8.8 \%$ y $1990=11.53 \%$; teniendo en cuenta que la pesca pasa de ser casi inexistente en 1970 a ocupar el 55\% del peso de este sector, y que en 1970 el $15.7 \%$ correspondía casi exclusivamente a ganadería. Hoy [año de elaboración del informe, con datos hasta 1991 y primer semestre de 1992] la ganadería no llega al 5\% del PBG y la pesca representa el 6.4\% del PBG" (UFPA, 1992).

Este señalamiento tiene por objeto poner en evidencia cómo ha variado la composición interna del sector AGYP, y no supone valoraciones sobre modificaciones estructurales en la economía satacruceña.

Asimismo, la caída de la productividad de la explotación ganadera es registrada en 1995, respecto de la participación sectorial en el PBG, con los siguientes números: "la participación del sector primario disminuye en el PBG del 45.3\% en 1988 a 42\% en 1992 [...] la evolución por rama de actividad muestra la caída de Agricultura, Ganadería y Pesca de un 187.5\% en 1988 a 178.1\% en 1992 (base $1980=100$ ). Siendo la única rama de actividad

${ }^{9}$ Mabel Manzanal (2000) menciona, respecto del PBG/capita, que: “(...) no es el mejor indicador para evaluar las desigualdades regionales (aunque también es cierto que no existe otro para expresar en síntesis esta realidad) [y agrega en nota al pie]: uno de los problemas de que adolece el $\mathrm{PBG} /$ capita es que no refleja la profunidad de las desigualdades socio-territoriales, en tanto es un promedio influido por los altos niveles de ingresos de la población del estrato superior, lo cual se acentúa en países fuertemente polarizados en su estructura social, como sucede con los latinoamericanos. Así mismo, no refleja adecuadamente la realidad en ámbitos con escasa población y enormes ingresos públicos (como sucede en zonas con recursos provenientes de regalías de la explotación petrolífera o con otros beneficios especiales)" (2000: 434). Si bien la referencia de este artículo es al PBG por sectores, es oportuna la aclaración, en tanto es sobre la base de éste que luego se calcula el PBG per cápita, lo que llevaría a sobredimensionar el impacto real de la redistribución de esos ingresos. 
que disminuyó su participación en el PBG en ese periodo" (UFPA/ UBA, 1995b: 5).

Puede suponerse que la caída no fue mayor para este sector en razón de la relevancia que venía adquiriendo el subsector pesca y a un incipiente crecimiento de la cría y venta de ganado bovino, así como también a la puesta en funcionamiento de cultivos bajo cubierta, sobre todo en el sur de la provincia y a la intensificación de la producción de fruta fina (frambuesa, frutilla y cerezas) en el noroeste de la provincia.

\section{Desertificación y monoproducción lanera}

La lana, producto básico de la explotación ovina, sufre desde hace años constantes fluctuaciones de su precio, lo que hace prácticamente imposible realizar algún tipo de previsión con base en los ingresos generados por su venta. En el área en estudio, a esas variaciones debe añadirse el hecho de que la lana que allí se produce no es en general de calidad superior, por lo que su precio todavía pierde un poco más con relación al que se paga por lanas finas. ${ }^{10}$

Respecto de la incidencia de la desertificación en la pérdida de ingresos, son escasos los estudios que vinculen directamente estas variables. No obstante, se estima que "en los últimos 30 años, el Ingreso Bruto no percibido por Chubut y Santa Cruz fue de 260 millones de dólares. La magnitud económica del deterioro es tal, que se calcula la pérdida de una zafra de lana completa cada 7 años" (LUDEPA, 1992: 35).

Es ilustrativa la apreciación realizada Rapp et al. (1998), relacionada con la composición de los ingresos en la mayoría de las explotaciones en Santa Cruz, que compara con dos de los mayores productores ovinos del mundo: "en Australia la relación de productos lana-carne resulta aproximadamente 60\% y $40 \%$ y en Nueva Zelanda del 50\% y 50\%. En Santa Cruz, si se considera el promedio general, difícilmente pueda superarse en la actualidad el $5 \%$ de ingresos por carne, correspondiendo a la lana el 95\%, aunque en muchas zonas de la provincia, desde hace varios años, la relación es 0\% carne y 100\% lana. Este desequilibrio conduce a una explotación irracional al no permitir las corres-

${ }^{10}$ Un elemento que puede contribuir a explicar una parte de la caída en los precios de la lana es el continuo reemplazo de este producto por fibra sintética, por lo que el mercado se reduce constantemente y la demanda mundial es cubierta mayormente por dos grandes productores ovinos: Nueva Zelanda y Australia. 
pondientes descargas de hacienda en los momentos más apropiados y ello es precisamente una de las causas de la sobrecarga animal" (Rapp et al., 1988: 156).

Respecto de la modalidad de venta de la lana, los productores de la zona mantienen en general la forma de comercialización tradicional: "el 75\% se vende al barrer sin tipificar; el 14\% al barrer tipificada y el 7\% sobre el lomo del animal. De acuerdo con datos del censo 1988, el 76.41\% no clasificaba la lana" (Barbería, 1995: 40). En este sentido, es necesario destacar que "el $60 \%$ de los establecimientos produce menos de 15,000 kg de lana sucia, que significan aproximadamente $8,000 \mathrm{~kg}$ de lana limpia (...) esto ha llevado a que muchos establecimientos de 20,000 has sean considerados minifundios por su nivel de rentabilidad. Esto es, no cubren las necesidades básicas con los ingresos del mismo" (Quargnolo y Álvarez, 1991).

Muchos de los establecimientos comprendidos dentro del $60 \%$ señalado se encontrarían en una situación muy comprometida; tal es así que un documento del INTA señala que "el 50\% del estrato de 0-1,000 ovinos [251 en la provincia en el año 1988; para el año 1991 había 108 en ese estrato], corresponde a establecimientos en una faz terminal de sus recursos, imposible de recuperar con la tecnología disponible” (INTA, 1993).

Las dos menciones anteriores señalan un camino muy difícil para muchas explotaciones: pueden seguir en producción mientras la tierra y el escaso pastizal natural resista el pastoreo y el pisoteo, no obstante, terminarán cerrando ante la imposibilidad de obtener la subsistencia por ese medio.

\section{Factibilidad de alternativas productivas}

La siguiente expresión refleja la situación actual de muchos establecimientos en la meseta central de Santa Cruz: "la receptividad ganadera actual no supera los 0.1 ovinos/ha (esto significa que se requieren 10 hectáreas por cada ovino) con lo cual los establecimientos no alcanzan a ser unidades económicamente sostenibles. Actualmente, sería necesario para ello un tamaño de 60,000 ha, lo que permitiría sostener unos 6,000 ovinos. Los establecimientos actuales, con un tamaño medio de 20,000 has, están condenados a sobrepastorear y depender de fuertes subsidios estatales [...] el uso pasturil del área sólo parece sostenible en las unidades de paisaje menos susceptibles, luego de una fuerte concentración de la propiedad [...] hay que posibilitar usos alternativos que no 
contemplen el modelo ganadero tradicional, ya agotado" (Oliva, 1992).

Desde esta óptica, la crisis es recurrente, porque es el sistema de producción el que se desajustó respecto de las potencialidades del recurso natural y, consecuentemente, ha perdido vigencia. Sería necesario reorientar el proceso productivo, ajustarlo a otros parámetros, donde la sustentabilidad, tanto económica, como ecológica y social sean la meta (Pickup y Stafford, 1993).

Una de las vías alternativas en los últimos años se centra en el agroturismo y el cultivo de ajo. La primera requiere que el establecimiento, además de su ubicación rural, tenga algún atractivo que ofrecer al turista, y la segunda variante requiere esencialmente disponer de agua y un cierto capital inicial, además de algunos conocimientos básicos acerca del cultivo de ese producto y, quizá más relevante aún, cierta disposición por parte del productor para iniciarse en tal actividad.

Para los que quieren continuar con la producción ovina, una tercera variante es realizar relevamientos de pastizal natural para ajustar la cantidad de animales a la disponibilidad de forraje. Por un lado, esto requiere no sólo dinero para realizar tal estudio, sino que tal vez resulte necesario hacer nuevas divisiones en el campo, lo cual resulta mucho más costoso. Por otro lado, considerando el estado del pastizal natural en el área de estudio, probablemente el número de ovinos que podría apacentar no permitirá al productor seguir viviendo de la explotación ganadera.

Al respecto, Barbería decía que: "recién en los últimos años - desde mediados de los 80 y con más intensidad desde los 90- se iniciaron relevamientos y estudios sobre el deterioro de los pastizales, sus posibilidades de recuperación y manejo adecuado. Además de muy recientes, requieren experimentación, financiación y un cambio de mentalidad del ganadero, que aún continúa atribuyendo las causas de la crisis a factores climáticos y a los precios de la lana; por otra parte, no cuentan con capital suficiente para invertir en tecnología, ya que prevalece el minifundio (establecimientos con menos de 5,000 ovinos), a excepción de la zona sur y de una estrecha franja costera que alcanza la localidad de Puerto San Julián” (Barbería, 1995: 281. Cursivas propias).

La expresión: "cambio de mentalidad del ganadero" es frecuente en las organizaciones técnicas y estudiosos dedicados al quehacer agropecuario. Quizá pueda ser la meta por lograr, eso hay que establecerlo, pero sí sostenemos que es necesario conocer cómo opera el pensamiento del productor, comprender qué 
elementos toma en consideración y cómo los articula para llegar a esas y no a otras ideas acerca de la producción y su comprensión del devenir de la situación actual. Sólo de ese modo se podrá abrigar la esperanza de un cambio en el futuro.

En un taller sobre aspectos relacionados con la desertificación leemos que "se porfía en volver a las cargas animales históricas, pero no en ajustarla a la condición actual del recurso para no repetir daños. El factor rentabilidad impide en muchos casos que se realice un ajuste de carga adecuado a la condición del pastizal" (LUDEPA, 1992. Cursivas propias).

Quizá en el estudio y comprensión de las actitudes porfiadas podamos encontrar pistas sólidas para implantar una estrategia de lucha concreta que tome en cuenta el punto de vista de los productores y, por consiguiente, logre su apoyo.

Con el fin de comprender el proceso de desertificación en su relación con el modo en que se dio la ocupación de las tierras en Santa Cruz, recupero de la obra de Barbería las expresiones que da cuenta de este último proceso: "la cantidad de potreros, el número y calidad de las viviendas, galpones de esquila y bañaderos van disminuyendo de sur a norte, en relación directa con el tamaño, forma de tenencia, disponibilidad de capital y receptividad de los lotes" (Barbería, 1995: 282). De un modo simple y casi superficial, puede establecerse entonces una asociación entre la forma y momento de la ocupación con el desarrollo y avance del proceso erosivo. Digo de modo simple y casi superficial, porque habría que profundizar en esa relación para no caer en generalizaciones que oculten las variaciones que pudieran existir.

\section{Evolución del stock ovino en los establecimientos del área de estudio}

El cuadro 1 permite observar una caída sostenida del stock ovino en los departamentos Magallanes y Río Chico, foco del estudio. El descenso es notorio aun en el departamento Guer Aike, ubicado en el sur de Santa Cruz, uno de los pocos de los que hoy puede decirse mantiene una producción ovina con cierto margen de rentabilidad y afectado por leves grados de desertificación. 


\section{Cuadro 1}

Existencias ovinas en los departamentos de la Provincia de Santa Cruz (por años, en miles de ovinos)

\begin{tabular}{|l|r|r|r|r|r|r|r|r|}
\hline \multicolumn{1}{|c|}{ Años de seguimiento } \\
\hline Déartamento & \multicolumn{1}{|c|}{1937} & \multicolumn{1}{c|}{1947} & \multicolumn{1}{c|}{1960} & \multicolumn{1}{c|}{1968} & \multicolumn{1}{c|}{1975} & \multicolumn{1}{c|}{1978} & 1988 & \multicolumn{1}{c|}{1992} \\
\hline Magallanes & $1^{\prime} 081,027$ & 844,736 & 715,461 & 731,929 & 649,494 & 735,623 & 376,827 & 269,827 \\
\hline Deseado & 796,209 & 593,092 & 536,674 & 546,561 & 411,262 & 415,499 & 272,298 & 228,195 \\
\hline Lago Argentino & $1^{\prime} 920,620$ & $1^{\prime} 835,123$ & $1^{\prime} 601,124$ & $1^{\prime} 530,731$ & $1^{\prime} 262,309$ & $1^{\prime} 393,593$ & 818,561 & 567,767 \\
\hline Corpen Aike & 862,424 & $1^{\prime} 038,637$ & $1^{\prime} 091,658$ & 916,135 & 818,229 & 779,977 & 530,620 & 366,167 \\
\hline Guer Aike & 619,032 & 737,918 & 691,623 & 700,526 & 563,917 & 626,207 & 444,030 & 393,762 \\
\hline $\begin{array}{l}\text { Lago Buenos } \\
\text { Aires }\end{array}$ & $1^{\prime} 798,874$ & $1^{\prime} 726,388$ & $1^{\prime} 693,929$ & $1^{\prime} 720,458$ & $1^{\prime} 677,887$ & $1^{\prime} 699,280$ & $1^{\prime} 161,028$ & $1^{\prime} 205,485$ \\
\hline Total ovinos: & $7^{\prime} 503,568$ & 445,618 & 706,836 & 768,128 & 635,541 & 634,397 & 486,112 & 311,156 \\
\hline
\end{tabular}

Fuente: Censos Agropecuarios Nacionales y Provinciales 1937, 1947, 1960, 1968, 1975, 1978, 1988 y 1992.

El cuadro 2 permite apreciar la caída global del stock ovino para los años 1993 y subsiguientes. Según la Encuesta Nacional Agropecuaria (ENA), el stock ovino del país para 1997 era de 13’197,800 cabezas. El aporte de las provincias patagónicas era, según datos para el mismo año, de 57.53\% del total nacional (INDEC, 1998: 1718). La misma encuesta, para el año 2000 señala que: “(...) las existencias de ganado ovino totalizaron 13,6 millones de cabezas. Las provincias patagónicas concentran el 62\% del total de ovinos, Buenos Aires el 13\% y Corrientes el 8\%" (INDEC, 2000: 7).

\section{Cuadro 2}

Comparación del stock ovino en Santa Cruz y resto del país (en millones de cabezas y variación porcentual, años 1993/1997)

\begin{tabular}{|c|c|c|c|c|c|c|c|c|c|}
\hline Provincia & 1993 & $\begin{array}{c}\text { Variac } \% \\
94 / 93\end{array}$ & 1994 & $\begin{array}{c}\text { Variac } \% \\
95 / 94\end{array}$ & 1995 & $\begin{array}{c}\text { Variac \% } \\
96 / 95\end{array}$ & 1996 & $\begin{array}{c}\text { Variac \% } \\
97 / 96\end{array}$ & 1997 \\
\hline Santa Cruz & $\begin{array}{l}3,447.3 \\
\end{array}$ & -17.91 & $2,829.8$ & -10.78 & $2,524.7$ & -20.04 & $2,018.7$ & -0.91 & $2,002.3$ \\
\hline Total país & $18,436.2$ & -8.21 & $16,922.6$ & -9.92 & $15,244.6$ & -6.14 & 14,308 & -7.76 & $13,197.8$ \\
\hline
\end{tabular}

Fuente: Tomado de INDEC (1998).

Obviamente, la caída del stock por departamentos encuentra su razón en el descenso del mismo en cada explotación. Todas las explotaciones muestran una significativa disminución en el número de ovinos para los distintos años de seguimiento, en algunos de ellos estrepitosa si se compara el stock al iniciar la producción con el disponible en el año 1981 y en los años 1991 y 1998. No obstante, en otras se aprecia un incremento constante del número de ovinos desde el inicio de la explotación y luego, al- 
canzada la cima, una caída, en algunos casos más acentuada y en otros menos grave, pero invariablemente presentan un número menor de animales en stock.

La magnitud de la pérdida de stock se aprecia al comparar la evolución en el número de ovinos, para cuatro periodos seleccionados y por estratos de stock ovino en los 39 productores relevados en meseta central de Santa Cruz, con eje en la localidad de Gobernador Gregores (véase el cuadro 3).

\section{Cuadro 3}

Evolución del stock por superficie, estrato de ovinos y años de seguimiento (39 establecimientos, zona centro de Santa Cruz, en número de establecimientos)

\begin{tabular}{|c|r|c|c|c|c|}
\hline Núm. de ovinos & Años & hasta 15,000 & $\begin{array}{c}\mathbf{1 5 , 0 0 1} \text { a } \\
\mathbf{2 0 , 0 0 0}\end{array}$ & $\mathbf{2 0 , 0 0 1}$ y más & Total establec. \\
\hline & Al comenzar* & 2 & 1 & 0 & 3 \\
\hline & 1981 & 0 & 0 & 1 & 1 \\
\hline Hasta 1,000 & 1991 & 3 & 2 & 0 & 5 \\
\hline & 1998 & 8 & 5 & 3 & 16 \\
\hline & Al comenzar & 4 & 4 & 0 & 8 \\
\hline $\mathbf{1 , 0 0 1}$ a 3,000 & 1981 & 5 & 3 & 0 & 8 \\
\hline & 1991 & 7 & 6 & 3 & 16 \\
\hline & 1998 & 1 & 7 & 2 & 10 \\
\hline & Al comenzar & 6 & 10 & 6 & 22 \\
\hline & 1981 & 8 & 13 & 5 & 26 \\
\hline 3,001 y más & 1991 & 3 & 6 & 4 & 13 \\
\hline & 1998 & 1 & 1 & 2 & 4 \\
\hline
\end{tabular}

* En algunos casos el año de inicio de la explotación coincide con el actual propietario y en otros, los actuales propietarios refieren las cifras históricas de animales, con base en registros o a información obtenida por otras fuentes.

Fuente: Datos y elaboración propios, 1998.

Un análisis con cierto detalle de los datos que esta tabla aporta resulta apropiado con el fin de mostrar la magnitud de la caída del stock y la regresión que sufren los establecimientos con más extensión de tierra hacia rebaños cada vez menores y, a la vez, el descenso en cada año del seguimiento de los estratos con mayor cantidad de ovinos a los de menor número de animales.

Obsérvese por ejemplo que el estrato de 3,001 y más ovinos que en 1981 tenía 26 productores ubicados allí, en 1998 sólo tiene cuatro; en tanto que el estrato de hasta mil ovinos que en 1981 tenía sólo un productor, en 1998 reúne a 16 de ellos.

Otra lectura interesante de esta tabla es la relacionada con el stock y el tamaño del establecimiento. En tal sentido, caben dos aclaraciones: primera, en la zona de estudio, el tamaño pre- 
dominante es hasta 20 mil ha y, segunda, aun en los establecimientos más grandes (las dos columnas del medio) el stock se ha contraido hacia los estratos menores; por ejemplo, de diez establecimientos que al comenzar la explotación tenían entre 15,001 y 20,000 hectáreas y 3,001 y más ovinos, en 1998 encontramos allí un establecimiento, en tanto el estrato de 1,001 a 3,000 presenta siete, y el de hasta 1,000 ovinos, cinco explotaciones, lo cual da una pauta de la evolución negativa del stock y, por lógica, de la caída de la rentabilidad de las explotaciones.

\section{La carga estimada por el productor como indicador de percepción del estado del ecosistema}

La compleja relación entre necesidades de subsistencia y explotación del pastizal natural se hace patente en las explicaciones acerca de la potencialidad de la base material de la ganadería extensiva de ovinos y las expectativas del ganadero.

Con el fin de profundizar en el análisis de esta compleja relación, he diseñado una estrategia de abordaje particular, en el marco de la cual conceptué la percepción del ganadero sobre la potencialidad de la base natural (esto es, cuántos animales estima que el campo puede sostener) como “carga potencial”. En la gráfica 1 se menciona como "estimados productor".

\section{Gráfica 1}

Comparación de la carga estimada y necesaria por el productor y calculada por INTA con stock 1998. Meseta Central, 23 establecimientos en miles de ovinos

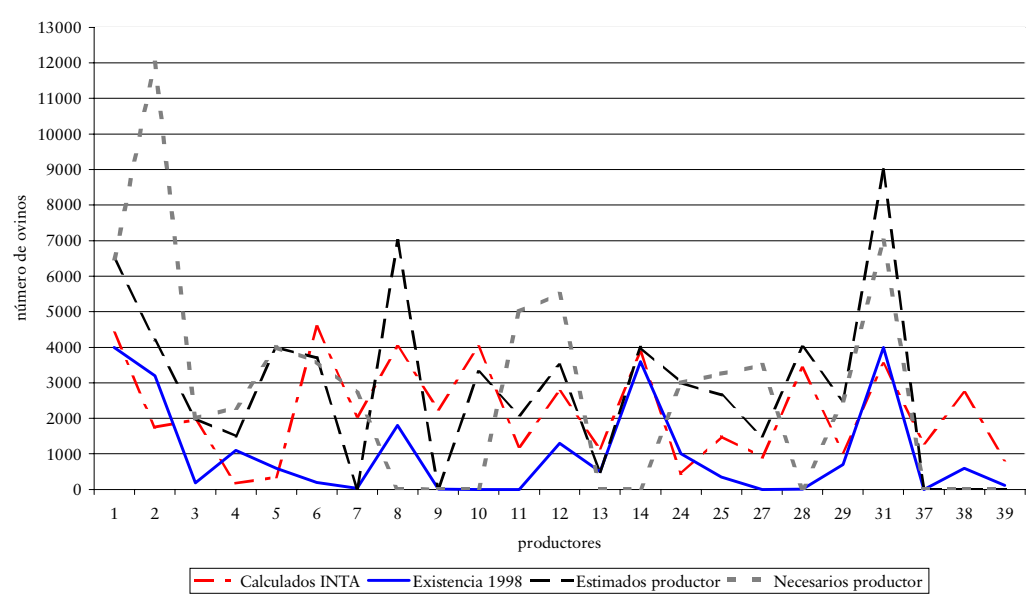


La importancia de emplear este constructo radica en que es posible compararlo con los resultados que emergen de estudios técnicos que, mediante el relevamiento de pastizales y la obtención de índices verdes (a través del procesamiento digital de fotos satelitales), estiman el número de animales que un determinado pastizal natural puede sostener. Esta variable es la que figura en la gráfica 1 como "calculados INTA".

La complejidad de la relación y su potencial impacto sobre el pastizal natural es evidente cuando se contrasta la diferencia entre "estimados productor" y "existencias 1998" (animales existentes en la explotación al momento de la encuesta).

El número de "estimados productor" asciende a 108,350 animales en los 39 encuestados, en tanto que las "existencias 1998 "son de 39,657 ovinos; y la diferencia entre lo que el productor posee actualmente y lo que estima que su campo podría matener es de 68,693 animales.

Por otro lado, el número de animales que los productores estiman necesarios para cubrir los gastos ("necesarios productor”) es de 118,050 ovinos. Entonces, si se compara el número de animales necesarios para cubrir sus gastos $(118,050)$ con el de “estimados productor" $(108,350)$, la diferencia es de apenas 9,700 animales.

Esta simple comparación permite apreciar uno de los modos en que opera el ganadero en su relación con el pastizal natural: el número de animales que el campo puede sostener, según su óptica, no surge de una estimación (vía relevamiento de pastizales, por ejemplo) de lo que el pastizal natural resistiría, sino que se acerca mucho (o coincide con él) al número de animales que estima necesario para cubrir sus necesidades.

De lo señalado, surgen dos cuestiones: primero, y según se aprecia en los relatos, existe entre los productores la conciencia de algunos elementos que dan cuenta de la crisis del ecosistema; no obstante, no son esos los factores relevantes en la explicación acerca del desencadenamiento de la situación actual. Para ser más claro: algunos productores aceptan que el sobrepastoreo es uno de los factores que llevaron a la situación actual, pero al explicar los problemas de su campo remiten constantemente a los bajos precios de la lana, cuestiones climáticas y depredares.

Segunda y más compleja, aunque la identificación de los elementos que desembocan en la situación actual pueda ser acertada, existen necesidades materiales concretas, relacionadas con la supervivencia que, sumadas a una necesidad de 'des-responsa- 
bilizarse' por el estado actual de las explotaciones, conjugan sus efectos y obliga a cargar nuevamente los campos, pese a la posible visión de su estado 'real'.

Con el fin de ajustar el estudio de la imagen que el productor tiene de la receptividad de su campo, dispongo de la "carga calculada" (número de animales que, según el relevamiento de pastizales, puede sostener el campo) por el INTA ${ }^{11}$ para 23 establecimientos del área estudiada, por lo que puede hacerse la comparación entre ésta y la asignada por el productor.

El relevamiento de pastizales es la técnica más simple y efectiva para determinar la producción de pasto por hectárea en cualquier campo. Es por ello que se toman los resultados de estos estudios, realizados en los campos cuyos productores fueron encuestados y se los compara con la carga potencial estimada por el productor.

Desde esta óptica, la visión que los productores tienen del entorno natural, en tanto sostén de la explotación ganadera extensiva que llevan adelante, es de extrema importancia, puesto que es en ese desajuste entre lo que el productor estima que puede hacer y lo que realmente el estado del suelo y del pastizal natural le permitiría realizar, donde la investigación indagó y pretendía hacer su aporte más relevante, haciendo evidente que no basta con 'mostrarle' al productor los efectos de un manejo no conservacionista del pastizal natural que ha llevado al estado actual a su explotación, sino que el productor mira y evalúa su campo desde otros parametros y considerando otros elementos, por ejemplo, el de remitir la carga actual a la comparación con la carga histórica o la de diez años atrás, con lo cual el campo aparece, en general, con menos animales y, por consecuencia, con más comida disponible para alimentar más ovinos.

La importancia de considerar la carga potencial como un indicador de la percepción del entorno natural por parte del productor reside en que la carga potencial depende del estado del suelo, en este caso, de la disponibilidad de pastizal natural para alimentar un número determinado de animales. El productor, según parece, no estima la carga potencial con ese criterio, sino que remite la misma a los stock ovinos en los años 1991, 1981, es decir

${ }^{11}$ El cálculo, dicen los autores, se realizó con base en la obtención de masas de índice verde (que relevan las existencias forrajeras) y mapas de unidades de paisaje (que permiten evaluar la aptitud del terreno para la cría de ovinos y, según la altura sobre el nivel de mar, también la magnitud del riesgo invernal y la posibilidad de realizar trabajos en campos diferenciados -invernada y veranada-) (Oliva, et al., s.f.). 
"a lo que siempre aguantó" y, como ya fue señalado, a la cantidad de animales que estima necesarios para cubrir sus gastos.

De tal modo que, al comparar la carga potencial con el stock de ovinos que dispone actualmente, surgen en los productores explicaciones que para dar cuenta de la caída sostenida del número de ovinos no toman en cuenta el deterioro del pastizal natural.

Puede apreciarse un esquema de la situación planteada en la gráfica 1. La lectura de la gráfica muestra que:

i. En general, el INTA estima una carga mayor (calculados INTA: línea y punto) de la que muchos productores tienen actualmente (existencias 1998: línea continua);

ii. La estimación del INTA no garantiza a la mayoría de los productores un número que pueda considerarse como una unidad económica rentable (necesarios productor: línea de puntos);

iii. Salvo excepciones, los productores pondrían más animales (estimados productor: línea de guiones) de los que estima el INTA;

iv. Las variaciones más notorias son las que se dan entre el número de animales que el productor estima necesarios y el número de ovinos disponibles en 1998.

Para los objetivos del estudio, era de fundamental interés captar los motivos y razones que sostenían (y sostienen) los productores para dar fundamento a su práctica de producción hoy. Era necesario ir más allá de lo que se ve, adentrarse en el campo más denso y complejo de los significados que orientan y dan sentido a la práctica productiva del ganadero.

Fue con esa intención que realicé entrevistas en profundidad con los productores. Al conversar sobre la evolución del stock, generalmente hacen hincapié en grandes catástrofes climáticas (gran sequía en 1983, nevadas y heladas en 1982 y 1984, la erupción del volcán Hudson en 1991, menor cantidad de lluvia y nieve en los últimos años) como causa de la caída del número de ovinos a valores realmente alarmantes.

Esas situaciones y elementos tienen una fuerte incidencia, aunque la explicación no se agota en ellos: "las agudas condiciones climáticas son sólo las causas desencadenantes del problema, pero su raíz se encuentra en el progresivo deterioro de los campos de pastoreo" (INTA Río Gallegos, 1986), a la vez que la “me- 
nor disponibilidad de forraje limita la producción en calidad y cantidad de lana” (Quargnolo y Álvarez, 1992).

Dos preguntas abiertas de la encuesta aplicada en la zona de estudio abordaban en términos generales la situación de evolución del stock ovino. Las respuestas dadas a la pregunta número 5 (¿cuáles son los problemas actuales de su campo?) y a la número 47 (¿ipor qué motivo no mantuvo el número de ovinos?), dieron la oportunidad de aproximarse a la visión que los productores tienen de esta situación.

Sobre 39 encuestas, las respuestas modales a la pregunta cinco fueron: treinta productores mencionan la incidencia de los depredadores como uno de los problemas actuales en sus explotaciones y trece señalan las inclemencias climáticas. El bajo precio de la lana obtiene doce menciones y el despoblamiento de campos, siete.

Al conversar sobre los motivos por los que no mantuvo el stock, para 29 respuestas la distribución es la siguiente: 18 mencionan la cuestión climática y 17 la incidencia de los depredadores.

Tanto en uno como en otro caso, las cuestiones climáticas y los depredadores emergen con importante peso en la explicación de la crisis. En ningún caso hubo referencias al sobrepastoreo como un elemento importante en el desencadenamiento de la situación actual.

\section{Reflexiones para seguir pensando}

Tal como fue expuesto, el deterioro del medio ambiente natural ha llevado a gran parte de la producción extensiva de ovinos en la provincia de Santa Cruz a una crisis que los organismos técnicos consideran terminal.

Buena parte de la crisis se asienta en dos circunstancias, cruciales en el largo plazo de la explotación: primera, la explotación a destajo del pastizal natural, las consecuencias de cuyo agotamiento son visibles actualmente, y segunda, la monoproducción lanera sin incorporación de valor agregado al producto final (recuérdese que aún hoy la lana se vende sin lavar, en el marco de un mercado internacional cada vez más exigente en cuanto a la calidad y presentación del producto).

El efecto combinado de estos dos elementos hace que, una vez agotada la posibilidad de extraer ganancia del producto absolutamente dominante en la mayoría de las explotaciones, los pro- 
ductores no encuentren una fuente genuina de ingresos para sostenerla y sostenerse.

Realicé también una aproximación que pretendió mostrar que las catástrofes climáticas no son la causa profunda de la crisis, sino el elemento visible que la agudizó, en tanto ésta venía gestándose desde el momento mismo que comenzó la explotación ovina extensiva en estas tierras: uso indiscriminado del pastizal natural, poca inversión y escasa o nula incorporación de tecnología.

La aspiración de éxito en la modificación de las prácticas productivas actuales debe contemplar las miradas de los productores, de lo contrario las propuestas de reconversión o manejo sustentable no recibirán el apoyo necesario.

Según el análisis del INTA y el Consejo Agrario Provincial, el camino más probable para aquellos que quieran seguir en producción es la reconversión productiva, que no es para todos y ello supone asumir riesgos y realizar inversiones que muchos no están dispuestos y otros no podrán realizar; en uno y otro caso quizá el cambio necesario más relevante sería el de convertirse de propietarios de campos a empresarios ganaderos, instancia ésta que complica aún más el panorama, en tanto la mayoría de las explotaciones, de lograr tal reconversión, no podrán seguir por la senda hasta ahora transitada en razón de la devastación del pastizal y suelo de los campos.

Otro factor que oscurece el encontrar una salida es el nivel de endeudamiento de estos productores, la avanzada edad de buena parte de ellos (los posibles continuadores de esta obra ya están radicados en las ciudades y con otras actividades) y el gran salto que supone convertirse en empresarios considerando la complejidad del mercado y el sistema bancario y financiero actuales.

La gravedad del deterioro exige, por lo menos en las zonas todavía viables productivamente, la puesta en marcha (o la potenciación si existiera) de un sistema de monitoreo permanente de la condición ecológica del pastizal y social de los productores, con el fin de evitar mayor deterioro del suelo y de la condición humana de quienes allí viven y laboran.

Esta afirmación supone también un seguimiento y asistencia de aquellos productores que actualmente sobreviven en las áreas más afectadas por la desertificación, procurando la puesta en práctica de medidas que promuevan su bienestar y no sólo atiendan al control y reversión del fenómeno ecológico. 


\section{Bibliografía}

Andrade, Larry (2002), Sociología de la desertificación en la Patagonia Austral: los productores ovinos de la Meseta Central de Santa Cruz, mimeo.

- (1999a), Representaciones sociales de la desertificación. El caso de los ganaderos ovino extensivos en la meseta central de Santa Cruz, Tesis de maestría inédita, Universidad Nacional Entre Ríos (UNER), Entre Ríos.

_ (1999b): "Ponencia en las Primeras Jornadas de Estudios Agrarios y Agroindustriales", Comisión Recursos Naturales y Sostenibilidad Ambiental, Facultad de Ciencias Económicas, noviembre, Universidad de Buenos Aires.

Atlas Barsa (1982), Enciclopedia Britannica, Inc.

Bertolani, Miguel A. (1989), "Ecología y desertificación en Patagonia”, Revista Patagonia Agropecuaria, año v, núm. 16, Patagonia.

Borelli, Pablo (s.f.), "Carga animal: un factor clave en el manejo de pastizales”, Presencia, año II, núm. 9.

Barbería, Elsa (1994), Crisis de la ganadería ovina en Santa Cruz. Sus causas, la intervención del Estado y de los empresarios, mimeo.

(1995), Los dueños de la tierra en la Patagonia Austral. 1880-1920, Universidad Federal de la Patagonia Austral, Patagonia.

Bendini, Mónica y Carlos Nogués (1992), Estudio sobre la percepción del proceso de desertificación que tienen los productores ganaderos, Provincia de Neuquen, GESA-Univ. del Comahue, mimeo.

Barton, Allen (1955), "Concepto de espacio de atributos en sociología”, en R. Boudon y P. Lazarfeld (1985), Metodología de las Ciencias Sociales, LAIA, Barcelona. 
Castro, Jose M. (s.f.), "Relevamiento de estados de erosión en la precordillera patagónica”, Presencia, año I, núm. 2.

Cepparo de Grosso, María E. (1986), La actividad pastoril en Santa Cruz: paisaje homogéneo, estructura invariable, Universidad Nacional de Cuyo, Mendoza.

Espina, Héctor (1994), “Área Piloto de Gobernador Gregores”, Proyecto LUDEPA, fase II, mimeo.

FLA (Federación Lanera Argentina) (1986), Producción lanera. Crisis y despegue, Buenos Aires.

Hugues, M., B. Griffon y C. Bouveyron (1978), Segmentación y tipología, Ed. Saltés, Madrid.

INTA (s.f.), Criterios para construir una tipología de productores en la zona centro de Santa Cruz, (s.p.i.) mimeo.

INTA Río Gallegos (1986), "Y cuando solo quede el viento", Presencia, año I, núm 2.

INTA (1993), Propuesta regional de reconversión productiva. Módulo Santa Cruz (preliminar), Centro Regional Patagonia Sur, EEA Santa Cruz, mimeo.

INTA (1997), Sistema soporte de decisiones. Un diagnóstico del estado, problemas y estrategias de desarrollo del sector agropecuario de la Patagonia Austral, PRODESAR, INTA/GTZ, Patagonia.

INTA Centro Regional Patagonia Sur (s.f.), página web: < http:// www.inta.gov.ar/crpatasu/prodesar/bariloche/ssd/rn $>$.

INDEC (1998), Encuesta Nacional Agropecuaria, Ministerio de Economía, Buenos Aires, página web: <http:// www.indec.mecon.gov.ar>

INDEC (2000), Encuesta Nacional Agropecuaria, Buenos Aires, página web: <http://www.indec.mecon.gov.ar>. 
Lafuente, Horacio Raúl (1981), La región de los Césares. Apuntes para una historia económica de Santa Cruz, Ed. de Belgrano, Buenos Aires.

LUDEPA (1992): Taller sobre aspectos socio-económicos y socioculturales relacionados con la desertificación en Patagonia, Bariloche, mimeo.

Manzanal, Mabel (2000), "Neoliberalismo y territorio en la Argentina de fin siglo" en Revista Economía, Sociedad y Territorio, vol. II, núm. 7, El Colegio Mexiquense, Zinacantepec, México, pp. 433-458.

Oliva, Gabriel (1992): Lucha contra la desertificación en Patagonia. Módulo Santa Cruz y Tierra del Fuego. Informe Ampliado, mimeo.

Oliva, Gabriel, Pablo Rial y Liliana González (s.f.), Mapa de desertificación en Santa Cruz. Transecta San Julián-Gobernador Gregores, Proyecto LUDEPA-SME, EEA INTA Río Gallegos, mimeo.

Panigatti, José Luis (1988), “Erosión”, en El deterioro del medio ambiente en la Argentina (suelo-agua-vegetación-fauna), FECIC, Buenos Aires.

Peralta, Carlos (1992), Marco Conceptual, Módulo Socio INTAGTZ, LUDEPA, Bariloche, mimeo.

Pickup, G. y Smith Stafford (1993), "Problems, prospect and procedures for assessing the sustainability of pastoral land management in arid Australia”, Journal of Biogeography 20, Australia.

Quargnolo, E. y R. Álvarez (1991), Zona centro: crisis de la producción ovina, mimeo.

Quargnolo, E. y R. Álvarez (1992): Zona Centro: crisis de la ganadería ovina, AER San Julián, mimeo.

Rapp, Emilia, Carlos Acosta y Rómulo Ayerza (1988), "Provincia de Santa Cruz", en El deterioro del medio ambiente en la 
Argentina (suelo-agua-vegetación-fauna), FECIC, Buenos Aires.

Schutz, Alfred (1995), El problema de la realidad social, Amorrortu Editores, Buenos Aires.

UFPA (Universidad Federal de la Patagonia Austral) (1992), Provincia de Santa Cruz. Estructura Poblacional, Río Gallegos, mimeo.

UFPA/UBA/MTSs (Universidad Federal de la Patagonia Austral, Universidad de Buenos Aires, Ministerio de Trabajo y Seguridad Social) (1994): "Primer Informe de Coyuntura Laboral 'Santa Cruz””, MTss, año I, núm. 1.

UfPA/Uba/MTss (Universidad Federal de la Patagonia Austral, Universidad de Buenos Aires, Ministerio de Trabajo y Seguridad Social) (1995a): Tercer Informe Laboral de la Provincia de Santa Cruz. Análisis de Coyuntura. Segundo Semestre 1994-Primer Semestre de 1995, mimeo.

UFPA/UBA (Universidad Federal de la Patagonia Austral, Universidad de Buenos Aires) (1995b): Análisis de Coyuntura. Segundo Semestre 1994-Primer Semestre 1995. Reestructuración Productiva y su Impacto Sobre el Empleo en PYMES, Microempresas y Cooperativas en mercados urbanos de la Provincia de Santa Cruz. Situación y Tendencias 91/95, mimeo.

UfPa/Uba/MTss (Universidad Federal de la Patagonia Austral, Universidad de Buenos Aires, Ministerio de Trabajo y Seguridad Social) (1995c): Primer Informe Especial de Coyuntura Ocupacional. Provincia de Santa Cruz. Demanda de Empleo y de Formación Profesional del Sector Privado Formal. Análisis Regionales, mimeo.

Weber, Max (1993), Ensayos sobre metodología sociológica, Amorrortu Editores, Buenos Aires. 